\title{
Dominating Sets and Domination Polynomials of Cycles
}

\author{
Saeid Alikhani ${ }^{a, b}$, and Yee-hock Peng ${ }^{b, c}$ \\ ${ }^{a}$ Department of Mathematics \\ Yazd University \\ 89195-741, Yazd, Iran \\ ${ }^{b}$ Institute for Mathematical Research, and \\ ${ }^{c}$ Department of Mathematics, \\ University Putra Malaysia, 43400 UPM Serdang, Malaysia
}

\begin{abstract}
Let $G=(V, E)$ be a simple graph. A set $S \subseteq V$ is a dominating set of $G$, if every vertex in $V \backslash S$ is adjacent to at least one vertex in $S$. Let $\mathcal{C}_{n}^{i}$ be the family of dominating sets of a cycle $C_{n}$ with cardinality $i$, and let $d\left(C_{n}, i\right)=\left|\mathcal{C}_{n}^{i}\right|$. In this paper, we construct $\mathcal{C}_{n}^{i}$, and obtain a recursive formula for $d\left(C_{n}, i\right)$. Using this recursive formula, we consider the polynomial $D\left(C_{n}, x\right)=\sum_{i=\left\lceil\frac{n}{3}\right\rceil}^{n} d\left(C_{n}, i\right) x^{i}$, which we call domination polynomial of cycles and obtain some properties of this polynomial.
\end{abstract}

Keywords: Dominating sets; Domination Polynomial; Recursive formula; Cycle

Mathematics subject classification: 05C69, 11B83

\section{Introduction}

Let $G=(V, E)$ be a simple graph of order $|V|=n$. A set $S \subseteq V$ is a dominating set of $G$, if every vertex in $V \backslash S$ is adjacent to at least one vertex in $S$. The domination number $\gamma(G)$ is the minimum cardinality of a dominating set in $G$. For a detailed treatment of this parameter, the reader is referred to [4]. It is well known and generally accepted that the problem of determining the dominating sets of an arbitrary graph is a difficult one (see [3]). Let $\mathcal{C}_{n}^{i}$ be the family of dominating sets of a cycle $C_{n}$ with cardinality $i$ and let $d\left(C_{n}, i\right)=\left|\mathcal{C}_{n}^{i}\right|$. We call the polynomial $D\left(C_{n}, x\right)=\sum_{i=\left\lceil\frac{n}{3}\right\rceil}^{n} d\left(C_{n}, i\right) x^{i}$, the domination

\footnotetext{
${ }^{1}$ Corresponding author. E-mail: alikhani@yazduni.ac.ir
} 
polynomial of cycle. For a detailed treatment of domination polynomial of a graph, the reader is referred to [1].

In the next section we construct the families of dominating sets of $C_{n}$ with cardinality $i$ by the families of dominating sets of $C_{n-1}, C_{n-2}$ and $C_{n-3}$ with cardinality $i-1$. We investigate the domination polynomial of cycle in Section 3.

As usual we use $\lceil x\rceil$, for the smallest integer greater than or equal to $x$. In this paper we denote the set $\{1,2, \ldots, n\}$ simply by $[n]$.

\section{Dominating sets of cycles}

Let $C_{n}, n \geq 3$, be the cycle with $n$ vertices $V\left(C_{n}\right)=[n]$ and $E\left(C_{n}\right)=\{(1,2),(2,3), \ldots,(n-$ $1, n),(n, 1)\}$. Let $\mathcal{C}_{n}^{i}$ be the family of dominating sets of $C_{n}$ with cardinality $i$. We shall investigate dominating sets of cycles. A simple path is a path where all its internal vertices have degree two. We need the following lemmas to prove our main results in this section:

Lemma 1. The following properties hold for cycles,

(i) $([2], p .364) \gamma\left(C_{n}\right)=\left\lceil\frac{n}{3}\right\rceil$.

(ii) $\mathcal{C}_{j}^{i}=\emptyset$, if and only if $i>j$ or $i<\left\lceil\frac{j}{3}\right\rceil$. (by (i) above).

(iii) If a graph $G$ contains a simple path of length $3 k-1$, then every dominating set of $G$ must contain at least $k$ vertices of the path. (by observation).

To find a dominating set of $C_{n}$ with cardinality $i$, we do not need to consider dominating sets of $C_{n-4}$ and $C_{n-5}$ with cardinality $i-1$. We show this in Lemma 2, Therefore, we only need to consider $\mathcal{C}_{n-1}^{i-1}, \mathcal{C}_{n-2}^{i-1}$, and $\mathcal{C}_{n-3}^{i-1}$. The families of these dominating sets can be empty or otherwise. Thus, we have eight combinations of whether these three families are empty or not. Two of these combinations are not possible (see Lemma $3(i)$ and (ii)). Also, the combination that $\mathcal{C}_{n-1}^{i-1}=\mathcal{C}_{n-2}^{i-1}=\mathcal{C}_{n-3}^{i-1}=\emptyset$; no need to be considered because it implies $\mathcal{C}_{n}^{i}=\emptyset$ (see Lemma $3($ iii $)$ ). Thus we only need to consider five combinations or cases. We consider this in Theorem 11. 
Lemma 2. If $Y$ is in $\mathcal{C}_{n-4}^{i-1}$ or $\mathcal{C}_{n-5}^{i-1}$ such that $Y \cup\{x\} \in \mathcal{C}_{n}^{i}$ for some $x \in[n]$, then $Y \in \mathcal{C}_{n-3}^{i-1}$.

Proof. Let $Y \in \mathcal{C}_{n-4}^{i-1}$ and $Y \cup\{x\} \in \mathcal{C}_{n}^{i}$ for some $x \in[n]$. This means, by Lemma 3 , we only need to consider $\{1, n-4\},\{2, n-4\}$ and $\{1, n-5\}$ as a subset of $Y$. In each case, $Y \in \mathcal{C}_{n-3}^{i-1}$. Now suppose that $Y \in \mathcal{C}_{n-5}^{i-1}$ and $Y \cup\{x\} \in \mathcal{C}_{n}^{i}$ for some $x \in[n]$. This means, by Lemma 3, $\{1, n-5\}$ must be a subset of $Y$. So $Y \in \mathcal{C}_{n-3}^{i-1}$.

The following lemma follows from Lemma 1(ii).

\section{Lemma 3.}

(i) If $\mathcal{C}_{n-1}^{i-1}=\mathcal{C}_{n-3}^{i-1}=\emptyset$, then $\mathcal{C}_{n-2}^{i-1}=\emptyset$,

(ii) If $\mathcal{C}_{n-1}^{i-1} \neq \emptyset$ and $\mathcal{C}_{n-3}^{i-1} \neq \emptyset$, then $\mathcal{C}_{n-2}^{i-1} \neq \emptyset$,

(iii) If $\mathcal{C}_{n-1}^{i-1}=\mathcal{C}_{n-2}^{i-1}=\mathcal{C}_{n-3}^{i-1}=\emptyset$, then $\mathcal{C}_{n}^{i}=\emptyset$.

The following lemma follow from Lemma 1(ii).

Lemma 4. Suppose that $\mathcal{C}_{n}^{i} \neq \emptyset$, then we have

(i) $\mathcal{C}_{n-1}^{i-1}=\mathcal{C}_{n-2}^{i-1}=\emptyset$, and $\mathcal{C}_{n-3}^{i-1} \neq \emptyset$ if and only if $n=3 k$ and $i=k$ for some $k \in N$,

(ii) $\mathcal{C}_{n-2}^{i-1}=\mathcal{C}_{n-3}^{i-1}=\emptyset$ and $\mathcal{C}_{n-1}^{i-1} \neq \emptyset$ if and only if $i=n$,

(iii) $\mathcal{C}_{n-1}^{i-1}=\emptyset, \mathcal{C}_{n-2}^{i-1} \neq \emptyset$ and $\mathcal{C}_{n-3}^{i-1} \neq \emptyset$ if and only if $n=3 k+2$ and $i=\left\lceil\frac{3 k+2}{3}\right\rceil$ for some $k \in N$,

(iv) $\mathcal{C}_{n-1}^{i-1} \neq \emptyset, \mathcal{C}_{n-2}^{i-1} \neq \emptyset$ and $\mathcal{C}_{n-3}^{i-1}=\emptyset$ if and only if $i=n-1$

(v) $\mathcal{C}_{n-1}^{i-1} \neq \emptyset, \mathcal{C}_{n-2}^{i-1} \neq \emptyset$ and $\mathcal{C}_{n-3}^{i-1} \neq \emptyset$ if and only if $\left\lceil\frac{n-1}{3}\right\rceil+1 \leq i \leq n-2$

\section{Proof.}


(i) $(\Rightarrow)$ Since $\mathcal{C}_{n-1}^{i-1}=\mathcal{C}_{n-2}^{i-1}=\emptyset$, by Lemma $1(i i)$, we have $i-1>n-1$ or $i-1<\left\lceil\frac{n-2}{3}\right\rceil$. If $i-1>n-1$, then $i>n$, and by Lemma $1(i i), \mathcal{C}_{n}^{i}=\emptyset$, a contradiction. So we have $i<\left\lceil\frac{n-2}{3}\right\rceil+1$, and since $\mathcal{C}_{n}^{i} \neq \emptyset$, together we have $\left\lceil\frac{n}{3}\right\rceil \leq i<\left\lceil\frac{n-2}{3}\right\rceil+1$, which give us $n=3 k$ and $i=k$ for some $k \in N$.

$(\Leftarrow)$ If $n=3 k$ and $i=k$ for some $k \in N$, then by Lemma 1( $i i)$, we have $\mathcal{C}_{n-1}^{i-1}=$ $\mathcal{C}_{n-2}^{i-1}=\emptyset$, and $\mathcal{C}_{n-3}^{i-1} \neq \emptyset$.

(ii) $(\Rightarrow)$ Since $\mathcal{C}_{n-2}^{i-1}=\mathcal{C}_{n-3}^{i-1}=\emptyset$, by Lemma 1 $(i i), i-1>n-2$ or $i-1<\left\lceil\frac{n-3}{3}\right\rceil$. If $i-1<\left\lceil\frac{n-3}{3}\right\rceil$, then $i-1<\left\lceil\frac{n-1}{3}\right\rceil$, and hence $\mathcal{C}_{n-1}^{i-1}=\emptyset$, a contradiction. So we must have $i>n-1$. Also since $\mathcal{C}_{n-1}^{i-1} \neq \emptyset$, we have $i-1 \leq n-1$. Therefore we have $i=n$.

$(\Leftarrow)$ If $i=n$, then by Lemma 11 $(i i)$, we have $\mathcal{C}_{n-2}^{i-1}=\mathcal{C}_{n-3}^{i-1}=\emptyset$ and $\mathcal{C}_{n-1}^{i-1} \neq \emptyset$.

(iii) $(\Rightarrow)$ Since $\mathcal{C}_{n-1}^{i-1}=\emptyset$, by Lemma $1(i i), i-1>n-1$ or $i-1<\left\lceil\frac{n-1}{3}\right\rceil$. If $i-1>n-1$, then $i-1>n-2$ and by lemma $1(i i), \mathcal{C}_{n-2}^{i-1}=\mathcal{C}_{n-3}^{i-1}=\emptyset$, a contradiction. So we must have $i<\left\lceil\frac{n-1}{3}\right\rceil+1$. But we also have $i-1 \geq\left\lceil\frac{n-2}{3}\right\rceil$ because $\mathcal{C}_{n-2}^{i-1} \neq \emptyset$. Hence, we have $\left\lceil\frac{n-2}{3}\right\rceil+1 \leq i<\left\lceil\frac{n-1}{3}\right\rceil+1$. Therefore $n=3 k+2$ and $i=k+1=\left\lceil\frac{3 k+2}{3}\right\rceil$ for some $k \in N$.

$(\Leftarrow)$ If $n=3 k+2$ and $i=\left\lceil\frac{3 k+2}{3}\right\rceil$ for some $k \in N$, then by Lemma $1(i i), \mathcal{C}_{n-1}^{i-1}=$ $\mathcal{C}_{3 k+1}^{k}=\emptyset, \mathcal{C}_{n-2}^{i-1} \neq \emptyset$ and $\mathcal{C}_{n-3}^{i-1} \neq \emptyset$.

(iv) $(\Rightarrow)$ Since $\mathcal{C}_{n-3}^{i-1}=\emptyset$, by Lemma $1(i i)$, we have $i-1>n-3$ or $i-1<\left\lceil\frac{n-3}{3}\right\rceil$. Since $\mathcal{C}_{n-2}^{i-1} \neq \emptyset$, by Lemma $1(i i)$, we have $\left\lceil\frac{n-2}{3}\right\rceil+1 \leq i \leq n-1$. Therefore $i-1<\left\lceil\frac{n-3}{3}\right\rceil$ is not possible. Hence we must have $i-1>n-3$. Thus $i=n-1$ or $n$. But $i \neq n$ because $\mathcal{C}_{n-2}^{i-1} \neq \emptyset$. So we have $i=n-1$.

$(\Leftarrow)$ If $i=n-1$, then by Lemma $1(i i), \mathcal{C}_{n-1}^{i-1} \neq \emptyset, \mathcal{C}_{n-2}^{i-1} \neq \emptyset$ and $\mathcal{C}_{n-3}^{i-1}=\emptyset$.

(v) $(\Rightarrow)$ Since $\mathcal{C}_{n-1}^{i-1} \neq \emptyset, \mathcal{C}_{n-2}^{i-1} \neq \emptyset$ and $\mathcal{C}_{n-3}^{i-1} \neq \emptyset$, then by applying Lemma 1( $\left.i i\right)$, we have $\left\lceil\frac{n-1}{3}\right\rceil \leq i-1 \leq n-1,\left\lceil\frac{n-2}{3}\right\rceil \leq i-1 \leq n-2$, and $\left\lceil\frac{n-3}{3}\right\rceil \leq i-1 \leq n-3$. So $\left\lceil\frac{n-1}{3}\right\rceil \leq i-1 \leq n-3$ and hence $\left\lceil\frac{n-1}{3}\right\rceil+1 \leq i \leq n-2$.

$(\Leftarrow)$ If $\left\lceil\frac{n-1}{3}\right\rceil+1 \leq i \leq n-2$, then by Lemma $1(i i)$, we have the result. 
The following theorem construct the families of dominating sets of $C_{n}$.

Theorem 1. For every $n \geq 4$ and $i \geq\left\lceil\frac{n}{3}\right\rceil$,

(i) If $\mathcal{C}_{n-1}^{i-1}=\mathcal{C}_{n-2}^{i-1}=\emptyset$ and $\mathcal{C}_{n-3}^{i-1} \neq \emptyset$, then

$$
\mathcal{C}_{n}^{i}=\mathcal{C}_{n}^{\frac{n}{3}}=\{\{1,4, \cdots, n-2\},\{2,5, \cdots, n-1\},\{3,6, \cdots, n\}\},
$$

(ii) If $\mathcal{C}_{n-2}^{i-1}=\mathcal{C}_{n-3}^{i-1}=\emptyset$ and $\mathcal{C}_{n-1}^{i-1} \neq \emptyset$, then $\mathcal{C}_{n}^{i}=\mathcal{C}_{n}^{n}=\{[n]\}$,

(iii) If $\mathcal{C}_{n-1}^{i-1}=\emptyset, \mathcal{C}_{n-2}^{i-1} \neq \emptyset$ and $\mathcal{C}_{n-3}^{i-1} \neq \emptyset$, then

$$
\begin{aligned}
& \mathcal{C}_{n}^{i}=\{\{1,4, \cdots, n-4, n-1\},\{2,5, \cdots, n-3, n\},\{3,6, \cdots, n-2, n\}\} \cup \\
& \left\{X \cup \left\{\begin{array}{ll}
\{n-2\}, \quad \text { if } 1 \in X \\
\left.\{n-1\}, \quad \text { if } 1 \notin X, 2 \in X \mid X \in \mathcal{C}_{n-3}^{i-1}\right\} \\
\{n\}, & \text { otherwise }
\end{array}\right.\right.
\end{aligned}
$$

(iv) If $\mathcal{C}_{n-3}^{i-1}=\emptyset, \mathcal{C}_{n-2}^{i-1} \neq \emptyset$ and $\mathcal{C}_{n-1}^{i-1} \neq \emptyset$, then $\mathcal{C}_{n}^{i}=\mathcal{C}_{n}^{n-1}=\{[n]-\{x\} \mid x \in[n]\}$,

(v) If $\mathcal{C}_{n-1}^{i-1} \neq \emptyset, \mathcal{C}_{n-2}^{i-1} \neq \emptyset$ and $\mathcal{C}_{n-3}^{i-1} \neq \emptyset$, then $\mathcal{C}_{n}^{i}=\left\{\{n\} \cup X \mid X \in \mathcal{C}_{n-1}^{i-1}\right\} \cup$

$$
\begin{aligned}
& \left\{X_{1} \cup\left\{\begin{array}{ll}
\{n\}, & \text { if } n-2 \text { or } n-3 \in X_{1}, \text { for } X_{1} \in \mathcal{C}_{n-2}^{i-1} \backslash \mathcal{C}_{n-1}^{i-1} \\
\{n-1\}, & \text { if } \left.n-2 \notin X_{1}, n-3 \notin X_{1} \text { or } X_{1} \in \mathcal{C}_{n-1}^{i-1} \cap \mathcal{C}_{n-2}^{i-1}\right\}
\end{array}\right\}\right. \\
& \left\{X_{2} \cup\left\{\begin{array}{ll}
\{n-2\}, & \text { if } 1 \in X_{2}, \text { for } X_{2} \in \mathcal{C}_{n-3}^{i-1} \text { or } X_{2} \in \mathcal{C}_{n-3}^{i-1} \cap \mathcal{C}_{n-2}^{i-1} \\
\{n-1\}, & \text { if } n-3 \in X_{2} \text { or } n-4 \in X_{2}, \text { for } X_{2} \in \mathcal{C}_{n-3}^{i-1} \backslash \mathcal{C}_{n-2}^{i-1}
\end{array}\right\} .\right.
\end{aligned}
$$

\section{Proof.}

(i) $\mathcal{C}_{n-1}^{i-1}=\mathcal{C}_{n-2}^{i-1}=\emptyset$ and $\mathcal{C}_{n-3}^{i-1} \neq \emptyset$. By Lemma $\llbracket(i), n=3 k, i=k$ for some $k \in N$. Therefore $\mathcal{C}_{n}^{i}=\mathcal{C}_{n}^{\frac{n}{3}}=\{\{1,4,7, \cdots, n-2\},\{2,5,8, \cdots, n-1\},\{3,6,9, \cdots, n\}\}$.

(ii) $\mathcal{C}_{n-2}^{i-1}=\mathcal{C}_{n-3}^{i-1}=\emptyset$ and $\mathcal{C}_{n-1}^{i-1} \neq \emptyset$. By Lemma $4(i i), i=n$. Therefore $\mathcal{C}_{n}^{i}=\mathcal{C}_{n}^{n}=\{[n]\}$.

(iii) $\mathcal{C}_{n-1}^{i-1}=\emptyset, \mathcal{C}_{n-2}^{i-1} \neq \emptyset$, and $\mathcal{C}_{n-3}^{i-1} \neq \emptyset$. By Lemma $4($ iii $), n=3 k+2, i=k+1$ for some $k \in N$. We denote the families $\{\{1,4, \cdots, 3 k-2,3 k+1\},\{2,5, \cdots, 3 k-1,3 k+$ 
$2\},\{3,6, \cdots, 3 k, 3 k+2\}\}$ and $\left\{X \cup\left\{\begin{array}{lll}\{3 k\}, & \text { if } 1 \in X \\ \{3 k+1\}, & \left.\text { if } 1 \notin X, 2 \in X \mid X \in \mathcal{C}_{3 k-1}^{k}\right\} \\ \{3 k+2\}, & \text { otherwise }\end{array}\right.\right.$ by $Y_{1}$ and $Y_{2}$, respectively. We shall prove that $\mathcal{C}_{3 k+2}^{k+1}=Y_{1} \cup Y_{2}$. Since $\mathcal{C}_{3 k}^{k}=$ $\{\{1,4,7, \cdots, 3 k-2\},\{2,5,8, \cdots, 3 k-1\},\{3,6,9, \cdots, 3 k\}\}$, then $Y_{1} \subseteq \mathcal{C}_{3 k+2}^{k+1}$. Also it is obvious that $Y_{2} \subseteq \mathcal{C}_{3 k+2}^{k+1}$. Therefore $Y_{1} \cup Y_{2} \subseteq \mathcal{C}_{3 k+2}^{k+1}$.

Now let $Y \in \mathcal{C}_{3 k+2}^{k+1}$, then by Lemma 1(iii), at least one of the vertices labeled $3 k+2,3 k+1$ or $3 k$ is in $Y$. Suppose that $3 k+2 \in Y$, then by Lemma 1( $(i i i)$, at least one of the vertices labeled 1,2 or 3 and $3 k+1,3 k$ or $3 k-1$ are in Y. If $3 k+1$ and at least one of $\{1,2,3\}$, and also $3 k$ and at least one of $\{1,2\}$ are in $Y$, then $Y-\{3 k+2\} \in \mathcal{C}_{3 k+1}^{k}$, a contradiction. If $\{3,3 k\}$ or $\{2,3 k-1\}$ is a subset of $Y$, then $Y=X \cup\{3 k+2\}$ for some $X \in \mathcal{C}_{3 k}^{k}$. Hence $Y \in Y_{1}$. If $\{1,3 k-1\}$ is a subset of $Y$, then $Y-\{3 k+2\} \in \mathcal{C}_{3 k+1}^{k}$, a contradiction. If $\{3,3 k-1\}$ is a subset of $Y$ and $\{3 k, 3 k+1\}$ is not a subset of $Y$, then $Y-\{3 k+2\} \in \mathcal{C}_{3 k-1}^{k}$. Hence $Y \in Y_{2}$. If $3 k+1$ or $3 k$ is in $Y$, we also have the result by the similar argument as above.

(iv) By Lemma $4(i v), i=n-1$. Therefore $\mathcal{C}_{n}^{i}=\mathcal{C}_{n}^{n-1}=\{[n]-\{x\} \mid x \in[n]\}$.

(v) $\mathcal{C}_{n-1}^{i-1} \neq \emptyset, \mathcal{C}_{n-2}^{i-1} \neq \emptyset$ and $\mathcal{C}_{n-3}^{i-1} \neq \emptyset$. First, suppose that $X \in \mathcal{C}_{n-1}^{i-1}$, then $X \cup\{n\} \in \mathcal{C}_{n}^{i}$. So $Y_{1}=\left\{\{n\} \cup X \mid X \in \mathcal{C}_{n-1}^{i-1}\right\} \subseteq \mathcal{C}_{n}^{i}$. Now suppose that $\mathcal{C}_{n-2}^{i-1} \neq \emptyset$. Let $X_{1} \in \mathcal{C}_{n-2}^{i-1}$. We denote $\left\{X_{1} \cup\left\{\begin{array}{ll}\{n\}, & \text { if } n-2 \text { or } n-3 \in X_{1}, \text { for } X_{1} \in \mathcal{C}_{n-2}^{i-1} \backslash \mathcal{C}_{n-1}^{i-1} \\ \{n-1\}, & \text { if } n-2 \notin X_{1}, n-3 \notin X_{1} \text { or } X_{1} \in \mathcal{C}_{n-1}^{i-1} \cap \mathcal{C}_{n-2}^{i-1}\end{array}\right\}\right.$ simply by $Y_{2}$. By Lemma 1(iii), at least one of the vertices labeled $n-3, n-2$ or 1 is in $X_{1}$. If $n-2$ or $n-3$ is in $X_{1}$, then $X_{1} \cup\{n\} \in \mathcal{C}_{n}^{i}$, otherwise $X_{1} \cup\{n-1\} \in \mathcal{C}_{n}^{i}$. Hence $Y_{2} \subseteq \mathcal{C}_{n}^{i}$. Here we shall consider $\mathcal{C}_{n-3}^{i-1} \neq \emptyset$. Let $X_{2} \in \mathcal{C}_{n-3}^{i-1}$. We denote $\left\{X_{2} \cup\left\{\begin{array}{l}\{n-2\}, \text { if } 1 \in X_{2} \text {, for } X_{2} \in \mathcal{C}_{n-3}^{i-1} \text { or } X_{2} \in \mathcal{C}_{n-3}^{i-1} \cap \mathcal{C}_{n-2}^{i-1} \\ \{n-1\}, \text { if } n-3 \in X_{2} \text { or } n-4 \in X_{2} \text {, for } X_{2} \in \mathcal{C}_{n-3}^{i-1} \backslash \mathcal{C}_{n-2}^{i-1}\end{array}\right\}\right.$, simply by $Y_{3}$. If $n-3$ or $n-4$ is in $X$, then $X \cup\{n-1\} \in \mathcal{C}_{n}^{i}$, otherwise $X_{2} \cup\{n-2\} \in \mathcal{C}_{n}^{i}$. Hence $Y_{3} \subseteq Y$. Therefore we've proved that $Y_{1} \cup Y_{2} \cup Y_{3} \subseteq \mathcal{C}_{n}^{i}$.

Now suppose that $Y \in \mathcal{C}_{n}^{i}$, so by Lemma 1(iii), $Y$ contain at least one of the vertices 
labeled $n, n-1$ or $n-2$. If $n \in Y$, so again by Lemma 1(iii) at least one of the vertices labeled $n-1, n-2$ or $n-3$ and 1,2 or 3 are in $Y$. If $n-2 \in Y$ or $n-3 \in Y$, then $Y=X \cup\{n\}$ for some $X \in \mathcal{C}_{n-2}^{i-1}$. Hence $Y \in Y_{2}$. Otherwise $Y=X \cup\{n-1\}$ for some $X \in \mathcal{C}_{n-2}^{i-1}$. Hence $Y \in Y_{2}$. If $n-1$ or $n-2$ is in $Y$, we also have the result by the similar argument as above.

By Theorem 1 we have the following theorem for $\left|\mathcal{C}_{n}^{i}\right|$.

Theorem 2. If $\mathcal{C}_{n}^{i}$ is the family of dominating set of $C_{n}$ with cardinality $i$, then

$$
\left|\mathcal{C}_{n}^{i}\right|=\left|\mathcal{C}_{n-1}^{i-1}\right|+\left|\mathcal{C}_{n-2}^{i-1}\right|+\left|\mathcal{C}_{n-3}^{i-1}\right| .
$$

Proof. We consider the five cases in Theorem 1, We rewrite Theorem 1 in the following form:

(i) If $\mathcal{C}_{n-1}^{i-1}=\mathcal{C}_{n-2}^{i-1}=\emptyset$ and $\mathcal{C}_{n-3}^{i-1} \neq \emptyset$, then $\mathcal{C}_{n}^{i}=$ $\left\{\{n-2\} \cup X_{1},\{n-1\} \cup X_{2},\{n\} \cup X_{3} \mid 1 \in X_{1}, 2 \in X_{2}, 3 \in X_{3}, X_{1}, X_{2}, X_{3} \in \mathcal{C}_{n-3}^{i-1}\right\}$,

(ii) If $\mathcal{C}_{n-2}^{i-1}=\mathcal{C}_{n-3}^{i-1}=\emptyset$ and $\mathcal{C}_{n-1}^{i-1} \neq \emptyset$, then $\mathcal{C}_{n}^{i}=\left\{\{n\} \cup X \mid X \in \mathcal{C}_{n-1}^{i-1}\right\}$,

(iii) If $\mathcal{C}_{n-1}^{i-1}=\emptyset, \mathcal{C}_{n-2}^{i-1} \neq \emptyset$ and $\mathcal{C}_{n-3}^{i-1} \neq \emptyset$, then $\mathcal{C}_{n}^{i}=\left\{\{n\} \cup X_{1},\{n-1\} \cup X_{2} \mid X_{1}, X_{2} \in \mathcal{C}_{n-2}^{i-1}, 1 \in X_{2}\right\} \cup$

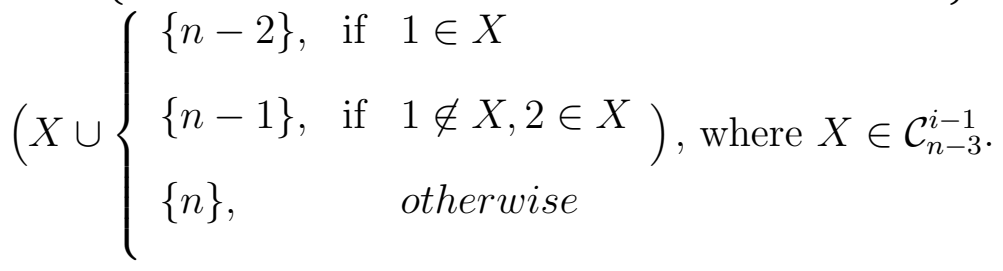

(iv) If $\mathcal{C}_{n-3}^{i-1}=\emptyset$ and $\mathcal{C}_{n-2}^{i-1} \neq \emptyset, \mathcal{C}_{n-1}^{i-1} \neq \emptyset$, then $\mathcal{C}_{n}^{i}=\left\{\{n\} \cup X_{1},\{n-1\} \cup X_{2} \mid X_{1} \in \mathcal{C}_{n-1}^{i-1}, X_{2} \in \mathcal{C}_{n-2}^{i-1}\right\}$.

(v) If $\mathcal{C}_{n-1}^{i-1} \neq \emptyset, \mathcal{C}_{n-2}^{i-1} \neq \emptyset$ and $\mathcal{C}_{n-3}^{i-1} \neq \emptyset$, then $\mathcal{C}_{n}^{i}=\left\{\{n\} \cup X \mid X \in \mathcal{C}_{n-1}^{i-1}\right\} \cup$ $\left\{X_{1} \cup\left\{\begin{array}{ll}\{n\}, & \text { if } n-2 \text { or } n-3 \in X_{1}, \text { for } X_{1} \in \mathcal{C}_{n-2}^{i-1} \backslash \mathcal{C}_{n-1}^{i-1} \\ \{n-1\}, & \text { if } n-2 \notin X_{1}, n-3 \notin X_{1} \text { or } X_{1} \in \mathcal{C}_{n-1}^{i-1} \cap \mathcal{C}_{n-2}^{i-1}\end{array}\right\} \cup\right.$ 
$\left\{X_{2} \cup \begin{cases}\{n-2\}, & \text { if } 1 \in X_{2}, \text { for } X_{2} \in \mathcal{C}_{n-3}^{i-1} \text { or } X_{2} \in \mathcal{C}_{n-3}^{i-1} \cap \mathcal{C}_{n-2}^{i-1} \\ \{n-1\}, & \left.\text { if } n-3 \in X_{2} \text { or } n-4 \in X_{2}, \text { for } X_{2} \in \mathcal{C}_{n-3}^{i-1} \backslash \mathcal{C}_{n-2}^{i-1}\right\} .\end{cases}\right.$ $X_{1} \in \mathcal{C}_{n-2}^{i-1} \backslash \mathcal{C}_{n-1}^{i-1}$ and $X_{2} \in \mathcal{C}_{n-3}^{i-1} \backslash \mathcal{C}_{n-2}^{i-1} \cap \mathcal{C}_{n-1}^{i-1}$.

By above construction, in every cases, we have $\left|\mathcal{C}_{n}^{i}\right|=\left|\mathcal{C}_{n-1}^{i-1}\right|+\left|\mathcal{C}_{n-2}^{i-1}\right|+\left|\mathcal{C}_{n-3}^{i-1}\right|$.

Since $\left|\mathcal{C}_{n}^{i}\right|$ satisfy the recursive formula with two variable, finding a formula for $\left|\mathcal{C}_{n}^{i}\right|$ is not easy. In the following theorem we use the generating function technique to find $\left|\mathcal{C}_{n}^{i}\right|$.

Theorem 3. For every natural $n \geq 4$ and $\left\lceil\frac{n}{3}\right\rceil \leq i \leq n,\left|\mathcal{C}_{n}^{i}\right|$ is the coefficient of $u^{n} v^{i}$ in the expansion of the function

$$
f(u, v)=\frac{u^{4} v^{2}\left(6+4 v+v^{2}+3 u+4 u v+u v^{2}+u^{2}+3 u^{2} v+u^{2} v^{2}\right)}{1-u v-u^{2} v-u^{3} v} .
$$

Proof. Set $f(u, v)=\sum_{n=4}^{\infty} \sum_{i=2}^{\infty}\left|\mathcal{C}_{n}^{i}\right| u^{n} v^{i}$. By recursive formula for $\left|\mathcal{C}_{n}^{i}\right|$ in Theorem 2 we can write $f(u, v)$ in the following form

$$
\begin{gathered}
f(u, v)=\sum_{n=4}^{\infty} \sum_{i=2}^{\infty}\left(\left|\mathcal{C}_{n-1}^{i-1}\right|+\left|\mathcal{C}_{n-2}^{i-1}\right|+\left|\mathcal{C}_{n-3}^{i-1}\right|\right) u^{n} v^{i}= \\
u v \sum_{n=4}^{\infty} \sum_{i=2}^{\infty}\left|\mathcal{C}_{n-1}^{i-1}\right| u^{n-1} v^{i-1}+u^{2} v \sum_{n=4}^{\infty} \sum_{i=2}^{\infty}\left|\mathcal{C}_{n-2}^{i-1}\right| u^{n-2} v^{i-1}+ \\
u^{3} v \sum_{n=4}^{\infty} \sum_{i=2}^{\infty}\left|\mathcal{C}_{n-3}^{i-1}\right| u^{n-3} v^{i-1}=u v\left(\left|\mathcal{C}_{3}^{1}\right| u^{3} v+\left|\mathcal{C}_{3}^{2}\right| u^{3} v^{2}+\left|\mathcal{C}_{3}^{3}\right| u^{3} v^{3}\right)+u v f(u, v)+ \\
+u^{2} v\left(\left|\mathcal{C}_{2}^{1}\right| u^{2} v+\left|\mathcal{C}_{2}^{2}\right| u^{2} v^{2}+\left|\mathcal{C}_{3}^{1}\right| u^{3} v+\left|\mathcal{C}_{3}^{2}\right| u^{3} v^{2}+\left|\mathcal{C}_{3}^{3}\right| u^{3} v^{3}\right)+u^{2} v f(u, v)+ \\
u^{3} v\left(\left|\mathcal{C}_{1}^{1}\right| u v+\left|\mathcal{C}_{2}^{1}\right| u^{2} v+\left|\mathcal{C}_{2}^{2}\right| u^{2} v^{2}+\left|\mathcal{C}_{3}^{1}\right| u^{3} v+\left|\mathcal{C}_{3}^{2}\right| u^{3} v^{2}+\left|\mathcal{C}_{3}^{3}\right| u^{3} v^{3}\right)+u^{3} v f(u, v)
\end{gathered}
$$

By substituting the values from Table 1, we have

$$
f(u, v)\left(1-u v-u^{2} v-u^{3} v\right)=u^{4} v^{2}\left(6+4 v+v^{2}+3 u+4 u v+u v^{2}+u^{2}+3 u^{2} v+u^{2} v^{2}\right)
$$

Therefore we have the result. 


\section{Domination polynomial of a cycle}

In this section we introduce and investigate the domination polynomial of cycles.

Definition 1. Let $\mathcal{C}_{n}^{i}$ be the family of dominating sets of a cycle $C_{n}$ with cardinality $i$ and let $d\left(C_{n}, i\right)=\left|\mathcal{C}_{n}^{i}\right|$. Then the domination polynomial $D\left(C_{n}, x\right)$ of $C_{n}$ is defined as

$$
D\left(C_{n}, x\right)=\sum_{i=\left\lceil\frac{n}{3}\right\rceil}^{n} d\left(C_{n}, i\right) x^{i} .
$$

By Definition 1 and Theorem 2, we have the following theorem.

Theorem 4. For every $n \geq 4$,

$$
D\left(C_{n}, x\right)=x\left[D\left(C_{n-1}, x\right)+D\left(C_{n-2}, x\right)+D\left(C_{n-3}, x\right)\right],
$$

with the initial values $D\left(C_{1}, x\right)=x, D\left(C_{2}, x\right)=x^{2}+2 x, D\left(C_{3}, x\right)=x^{3}+3 x^{2}+3 x$.

Using Theorem 2, we obtain the coefficients of $D\left(C_{n}, x\right)$ for $1 \leq n \leq 16$ in Table 1 . Let $d\left(C_{n}, j\right)=\left|\mathcal{C}_{n}^{j}\right|$. There are interesting relationships between the numbers $d\left(C_{n}, j\right)\left(\frac{n}{3} \leq\right.$ $j \leq n)$ in the table 1.

\begin{tabular}{r|rrrrrrrrrrrrrrrr}
$j$ & 1 & 2 & 3 & 4 & 5 & 6 & 7 & 8 & 9 & 10 & 11 & 12 & 13 & 14 & 15 & 16 \\
\hline$n$ & & & & & & & & & & & & & & & & \\
1 & 1 & & & & & & & & & & & & & & \\
2 & 2 & 1 & & & & & & & & & & & & & \\
3 & 3 & 3 & 1 & & & & & & & & & & & & \\
4 & 0 & 6 & 4 & 1 & & & & & & & & & & & \\
5 & 0 & 5 & 10 & 5 & 1 & & & & & & & & & & \\
6 & 0 & 3 & 14 & 15 & 6 & 1 & & & & & & & & & \\
7 & 0 & 0 & 14 & 28 & 21 & 7 & 1 & & & & & & & & \\
8 & 0 & 0 & 8 & 38 & 48 & 28 & 8 & 1 & & & & & & & \\
9 & 0 & 0 & 3 & 36 & 81 & 75 & 36 & 9 & 1 & & & & & & \\
10 & 0 & 0 & 0 & 25 & 102 & 150 & 110 & 45 & 10 & 1 & & & & & \\
11 & 0 & 0 & 0 & 11 & 99 & 231 & 253 & 154 & 55 & 11 & 1 & & & & \\
12 & 0 & 0 & 0 & 3 & 72 & 282 & 456 & 399 & 208 & 66 & 12 & 1 & & & \\
13 & 0 & 0 & 0 & 0 & 39 & 273 & 663 & 819 & 598 & 273 & 78 & 13 & 1 & & & \\
14 & 0 & 0 & 0 & 0 & 14 & 210 & 786 & 1372 & 1372 & 861 & 350 & 91 & 14 & 1 & & \\
15 & 0 & 0 & 0 & 0 & 3 & 125 & 765 & 1905 & 2590 & 2178 & 1200 & 440 & 105 & 15 & 1 & \\
16 & 0 & 0 & 0 & 0 & 0 & 56 & 608 & 2214 & 4096 & 4560 & 3312 & 1628 & 544 & 120 & 16 & 1
\end{tabular}

Table 1. $d\left(C_{n}, j\right)$ The number of dominating sets of $C_{n}$ with cardinality $j$.

In the following theorem, we obtain some properties of $d\left(C_{n}, j\right)$ : 
Theorem 5. The following properties hold for coefficients of $D\left(C_{n}, x\right)$ :

(i) For every $n \in N, d\left(C_{3 n}, n\right)=3$,

(ii) For every $n \geq 4, j \geq\left\lceil\frac{n}{3}\right\rceil, d\left(C_{n}, j\right)=d\left(C_{n-1}, j-1\right)+d\left(C_{n-2}, j-1\right)+d\left(C_{n-3}, j-1\right)$,

(iii) For every $n \in N, d\left(C_{3 n+2}, n+1\right)=3 n+2$,

(iv) For every $n \in N, d\left(C_{3 n+1}, n+1\right)=\frac{n(3 n+7)+2}{2}$,

(v) For every $n \geq 3, d\left(C_{n}, n\right)=1$,

(vi) For every $n \geq 3, d\left(C_{n}, n-1\right)=n$,

(vii) For every $n \geq 3, d\left(C_{n}, n-2\right)=\frac{(n-1) n}{2}$,

(viii) For every $n \geq 4, d\left(C_{n}, n-3\right)=\frac{(n-4)(n)(n+1)}{6}$,

(ix) for every $j \geq 4, \sum_{i=j}^{3 j} d\left(C_{i}, j\right)=3 \sum_{i=j-1}^{3 j-3} d\left(C_{i}, j-1\right)$,

(x) for every $n \geq 3,1=d\left(C_{n}, n\right)<d\left(C_{n+1}, n\right)<d\left(C_{n+2}, n\right)<\cdots<d\left(C_{2 n-1}, n\right)<$ $d\left(C_{2 n}, n\right)>d\left(C_{2 n+1}, n\right)>\cdots>d\left(C_{3 n-1}, n\right)>d\left(C_{3 n}, n\right)=3$.

(xi) If $S_{n}=\sum_{j=\left\lceil\frac{n}{3}\right\rceil}^{n} d\left(C_{n}, j\right)$, then for every $n \geq 4, S_{n}=S_{n-1}+S_{n-2}+S_{n-3}$ with initial values $S_{1}=1, S_{2}=3$ and $S_{3}=7$.

\section{Proof.}

(i) Since $\mathcal{C}_{n}^{3 n}=\{\{1,4,7, \ldots, 3 n-2\},\{2,5,8, \ldots, 3 n-1\},\{3,6,9, \ldots, 3 n\}\}$, so $d\left(C_{3 n}, n\right)=3$.

(ii) It follows from Theorem 2 ,

(iii) By induction on $n$. The result is true for $n=1$, because $\mathcal{C}_{2}^{5}=\{\{1,3\},\{1,4\},\{2,4\},\{2,5\},\{3,5\}\}$. Now suppose that the result is true for all natural numbers less than $n$, and we prove it for $n$. By $(i),(i i)$ and induction hypothesis, we have

$$
\begin{aligned}
d\left(C_{3 n+2}, n+1\right) & =d\left(C_{3 n+1}, n\right)+d\left(C_{3 n}, n\right)+d\left(C_{3 n-1}, n\right) \\
& =3 n+2 .
\end{aligned}
$$


(iv) By induction on $n$. Since $\mathcal{C}_{2}^{4}=\{\{1,2\},\{1,3\},\{1,4\},\{2,3\},\{2,4\},\{3,4\}\}$, so $d\left(C_{4}, 2\right)=6$, the result is true for $n=1$. Now suppose that the result is true for all natural numbers less than $n$, and we prove it for $n$. By $(i),(i i),(i i i)$ and induction hypothesis, we have

$$
\begin{aligned}
d\left(C_{3 n+1}, n+1\right) & =d\left(C_{3 n}, n\right)+d\left(C_{3 n-1}, n\right)+d\left(C_{3 n-2}, n\right) \\
& =3+3(n-1)+2+\frac{(n-1)(3(n-1)+7)+2}{2} \\
& =\frac{n(3 n+7)+2}{10} .
\end{aligned}
$$

(v) Since for any graph with $n$ vertices, $d(G, n)=1$, then we have the result.

(vi) Since for any graph with $n$ vertices, $d(G, n-1)=n$, we have the result.

(vii) By induction on $n$. The result is true for $n=3$, since $d\left(C_{3}, 1\right)=3$. Suppose that the result is true for all natural number less than $n$, and we prove it for $n$. By parts $(i i),(v),(v i)$ and induction hypothesis have

$$
\begin{aligned}
d\left(C_{n}, n-2\right) & =d\left(C_{n-1}, n-3\right)+d\left(C_{n-2}, n-3\right)+d\left(C_{n-3}, n-3\right) \\
& =\frac{(n-2)(n-1)}{2}+n-2+1 \\
& =\frac{(n-1) n}{2}
\end{aligned}
$$

(viii) By induction on $n$. The result is true for $n=4$, since $d\left(C_{4}, 1\right)=0$. Suppose that the result is true for all natural number less than $n$ and prove it for $n$. By parts (ii), (vi), (vii) and induction hypothesis we have

$$
\begin{aligned}
d\left(C_{n}, n-3\right) & =d\left(C_{n-1}, n-4\right)+d\left(C_{n-2}, n-4\right)+d\left(C_{n-3}, n-4\right) \\
& =\frac{(n-5)(n-1) n}{6}+\frac{(n-2)(n-3)}{2}+n-3 \\
& =\frac{(n-4) n(n+1)}{6}
\end{aligned}
$$

(ix) Proof by induction on $j$. First, suppose that $j=3$. Then $\sum_{i=3}^{9} d\left(C_{i}, 3\right)=54=$ $3 \sum_{i=2}^{6} d\left(C_{i}, 2\right)$. Now suppose that the result is true for every $j<k$, and we prove 
for $j=k$ :

$$
\begin{aligned}
\sum_{i=k}^{3 k} d\left(C_{i}, k\right) & =\sum_{i=k}^{3 k} d\left(C_{i-1}, k-1\right)+\sum_{i=k}^{3 k} d\left(C_{i-2}, k-1\right)+\sum_{i=k}^{3 k} d\left(C_{i-3}, k-1\right) \\
& =3 \sum_{i=k-1}^{3(k-1)} d\left(C_{i-1}, k-2\right)+3 \sum_{i=k-1}^{3(k-1)} d\left(C_{i-2}, k-2\right) \\
& +3 \sum_{i=k-1}^{3(k-1)} d\left(C_{i-3}, k-2\right)=3 \sum_{i=k-1}^{3 k-3} d\left(C_{i}, k-1\right) .
\end{aligned}
$$

(x) We shall prove that for every $n, d\left(C_{i}, n\right)<d\left(C_{i+1}, n\right)$ for $n \leq i \leq 2 n-1$, and $d\left(C_{i}, n\right)>d\left(C_{i+1}, n\right)$ for $2 n \leq i \leq 3 n-1$. We prove the first inequality by induction on $n$. The result hold for $n=3$. Suppose that result is true for all $n \leq k$. Now we prove it for $n=k+1$, that is $d\left(C_{i}, k+1\right)<d\left(C_{i+1}, k+1\right)$ for $k+1 \leq i \leq 2 k+1$.

By Theorem 2 and induction hypothesis we have

$$
\begin{aligned}
d\left(C_{i}, k+1\right) & =d\left(C_{i-1}, k\right)+d\left(C_{i-2}, k\right)+d\left(C_{i-3}, k\right) \\
& <d\left(C_{i}, k\right)+d\left(C_{i-1}, k\right)+d\left(C_{i-2}, k\right) \\
& =d\left(C_{i+1}, k+1\right)
\end{aligned}
$$

Similarly, we have the other inequality.

(xi) By Theorem 2, we have

$$
\begin{aligned}
S_{n} & =\sum_{j=\left\lceil\frac{n}{3}\right\rceil}^{n} d\left(C_{n}, j\right)=\sum_{j=\left\lceil\frac{n}{3}\right\rceil}^{n}\left(d\left(C_{n-1}, j-1\right)+d\left(C_{n-2}, j-1\right)+d\left(C_{n-3}, j-1\right)\right) \\
& =\sum_{j=\left\lceil\frac{n}{3}\right\rceil-1}^{n-1} d\left(C_{n-1}, j\right)+\sum_{j=\left\lceil\frac{n}{3}\right\rceil-1}^{n-2} d\left(C_{n-2}, j\right)+\sum_{j=\left\lceil\frac{n}{3}\right\rceil-1}^{n-3} d\left(C_{n-3}, j-1\right) \\
& =S_{n-1}+S_{n-2}+S_{n-3} .
\end{aligned}
$$

\section{References}

[1] Saeid Alikhani and Yee-hock Peng, Introduction to domination polynomial of a graph, to appear in Ars Combinatoria. 
[2] Gray Chartrand, Ping Zhang, Introduction to Graph Theory, Mc Graw Hill, Higher Education, 2005.

[3] M. R. Garey and D. S. Johnson, Computers and Intractability: A Guide to the Theorey of NP-Completness. Freeman, New York, 1979.

[4] T.W. Haynes, S.T. Hedetniemi, P.J. Slater, Fundamentals of Domination in Graphs, Marcel Dekker, NewYork, 1998. 